\title{
Hunting glycoproteins
}

Glycosylation is a feature of several of the proteins that are involved in the pathogenesis of Alzheimer's disease (AD), and aberrant glycosylation probably contributes to the generation of amyloid plaques and neurofibrillary tangles in the brain. To investigate this phenomenon, Huang and colleagues have set out to characterize all of the main brain glycoproteins, and their initial findings were recently reported in the European Journal of Neuroscience. Although this study has not yet uncovered any defects in the glycosylation process itself, the researchers did identify one important glycoprotein that was upregulated in $\mathrm{AD}$.

Huang et al. used affinity chromatography to isolate glycoproteins from post-mortem cerebral cortical tissue. The glycoproteins were separated using SDS-polyacrylamide gel electrophoresis and analysed by mass spectrometry. Eleven glycoproteins were isolated, one of which - the $\beta$-subunit of the lysosomal enzyme acid ceramidase showed increased expression levels and activity in the brains of individuals with AD.

The authors next compared the distribution of acid ceramidase in brain tissue from control individuals and patients with AD. In both cases, the enzyme was expressed in most neurons and some astrocytes, and in the tissue from individuals with $\mathrm{AD}$ it showed marked co-localization with the intracellular neurofibrillary tangles. This might imply that acid ceramidase is somehow involved in neurofibrillary degeneration. However, its increased expression and activity could also be a protective response to $\mathrm{AD}$-associated ceramide accumulation in the brain. Ceramide induces neuronal apoptosis, but acid ceramidase catalyses its conversion to sphingosine-1-phosphate - an antiapoptotic agent.

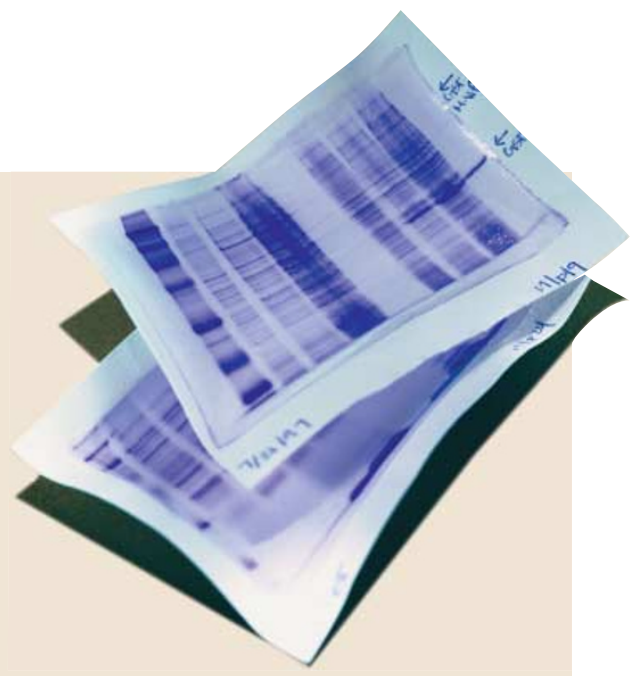

Further studies will be required to determine the true significance of the elevated acid ceramidase levels. It will also be interesting to analyse the other glycoproteins that were isolated in this screen to find out whether any of them show defects in glycosylation in the brains of individuals with $\mathrm{AD}$.

Heather Wood

\section{(2) References and links}

ORIGINAL RESEARCH PAPER Huang, Y. et al. Elevation in the level and activity of acid ceramidase in Alzheimer's disease brain. Eur. J. Neurosci. 20, 3489-3497 (2004)

\section{MEMORY THEORY}

\section{Crossing the divide}

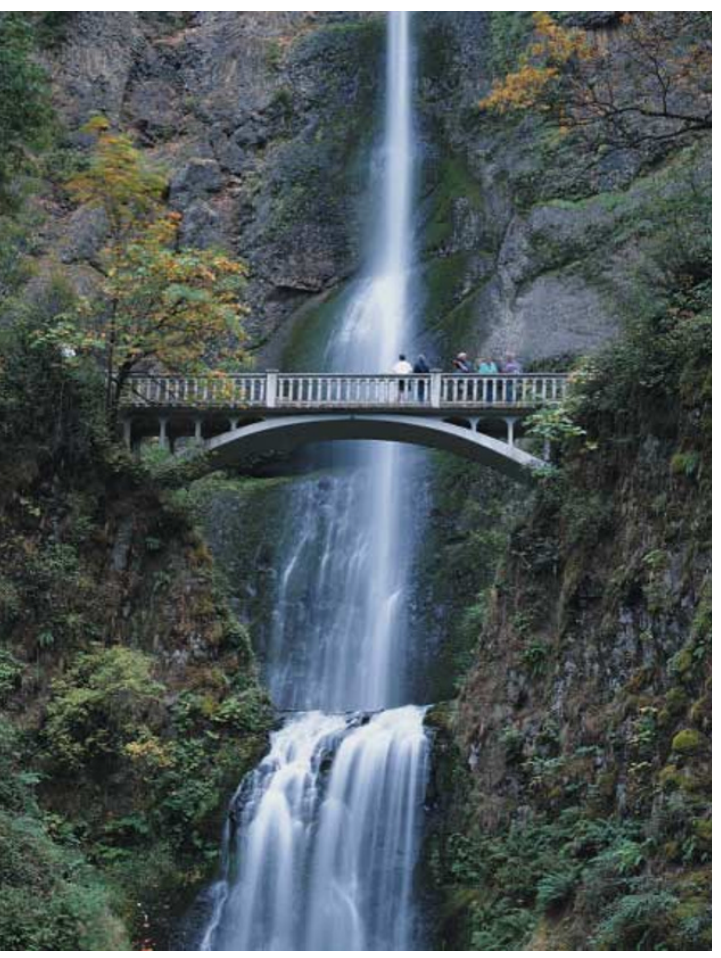

There are two types of memory: explicit (declarative) memory, which concerns facts and experiences, can be recalled by conscious effort and reported verbally, whereas implicit (nondeclarative) memory, which involves skill learning, cannot. However, new findings by Park and colleagues indicate that this dichotomous distinction might be oversimplistic. In a double-blind study, they show that midazolam — a drug that was thought to impair only explicit memory impairs implicit memory in healthy volunteers.

Early neuropsychological studies showed that patients with amnesia were impaired on explicit, but not implicit, memory tasks, which was thought to indicate that the two types of memory use different regions of the brain. However, this was called into question when later studies showed that some patients with amnesia also had deficits in a form of implicit memory known as contextual cuing. Contextual cuing is an implicit process that results in improved performance in spatial configuration tests, such as a visual search task, when display patterns are repeated.

Results from neuropsychological studies are unlikely to be conclusive because the patterns of brain damage between patients can vary considerably. In this new study, 27 healthy participants were tested for implicit memory using a visual task after they had been injected with either saline or midazolam. Each participant was tested under both conditions, so that each served as his or her own control. The participants performed better over time after both treatments, which is consistent with the literature on skill learning. The drug did not affect performance in the first trial, but the participants showed less facilitation for repeated displays in subsequent trials after injection with midazolam. They seemed to have difficulty learning the association between pieces of information, and so took a significantly longer time to identify the targets under midazolam when the repeated configurations cued the location of the targets.

Based on these findings, the authors suggest that conscious accessibility should not be the only criteria for defining different memory systems. Instead, the information processing requirements of a task, such as associative processing (or binding) of cues and context, might be an alternative framework for separating different types of memory performance. If memories require binding — whether explicit or implicit - then they are subject to amnesia.

Whether this new conception of memory is correct has yet to be determined. However, it is clear that the distinction between explicit and implicit memory is not as clear cut as it first seemed.

Jane Qiu

(2) References and links

ORIGINAL RESEARCH PAPER Park, $\mathrm{H}$. et al. The effect of midazolam on visual search: implications for understanding amnesia. Proc. Natl Acad. Sci. USA 101, 17879-17883 (2004) FURTHER READING Millin, P. M. et al. Interpretations of retrograde amnesia: old problems redux. Nature Rev. Neurosci. 2, 68-70 (2001) 